\title{
Efficient coupling of free propagating light into Whispering Gallery Modes
}

\author{
Xavier Zambrana-Puyalto ${ }^{1,2, *}$, Davide D’ Ambrosio ${ }^{2}$, and Gianluca Gagliardi² \\ ${ }^{1}$ Istituto Italiano di Tecnologia, Via Morego 30, 16136 Genova, Italy \\ ${ }^{2}$ Consiglio Nazionale delle Ricerche, Istituto Nazionale di Ottica (INO), via Campi Flegrei 34-Comprensorio A. Olivetti, 80078 \\ Pozzuoli (Na), Italy
}

\begin{abstract}
Whispering Gallery Mode resonators are dielectric structures with cylindrical symmetry. They are typically excited with an evanescent field leaking out of a tapered fiber or a waveguide. It is also known that they can be excited with free propagating beams. In this work, we use a recently developed analytical model which quantitatively describes the coupling of free propagating beams into Whispering Gallery Modes for spherical particles. Using this model, we have been able to theoretically quantify the mode purity and the coupling efficiency of a resonant Whispering Gallery Mode of an order $j^{*}=1456$. We have observed that the transverse position of the beam plays a crucial role in determining the mode purity and coupling efficiency. Last but not least, we have verified that the coupling efficiency as well as the Q-factor predicted by our model are in an outstanding agreement with the experimental values measured on a microresonator of the same dimensions as the simulated one.
\end{abstract}

\section{Introduction}

Whispering Gallery Mode (WGM) resonators are dielectric structures with cylindrical symmetry. Their sizes tend to range from ten to a few hundreds of micrometers. These optical resonators have incredibly high $\mathrm{Q}$ factors and their use is widely spread across the photonic sciences $[1,2]$. Most of the WGM resonators are excited via evanescent coupling, yet it has also been demonstrated that they can be excited via direct scattering [3,4]. Recently, we have theoretically demonstrated that the WGM excitation in direct scattering set-ups is due to angular momentum (AM) matching [5]. We showed that the AM content of the WGMs induced in a spherical microresonator must match the AM content of the excitation beam. To do that, we used the analytical Generalized Mie Theory [6, 7] in the transverse angular momentum (TAM) basis [8]. The choice of the TAM basis is critical in order to be able to simulate particles with diameters greater than $100 \mu \mathrm{m}$ [5] at optical frequencies. Here, we focus our attention on the mode purity (MP) and the coupling efficiency (CE) of the excited WGMs. Moreover, we compare our theoretical predictions with the experimental data collected for a microdroplet resonator with a diameter of $300 \mu \mathrm{m}$. As it will be shown, the match of our theoretical and experimental data is outstanding, not only in terms of CE, but also in terms of resonance spectrum, Q-factor of resonances, and EM field distribution.

\section{Theoretical model}

Our theoretical model is developed within the Mie Theory framework. That is, we consider an isotropic, ho-

*e-mail: xavislow@protonmail.com

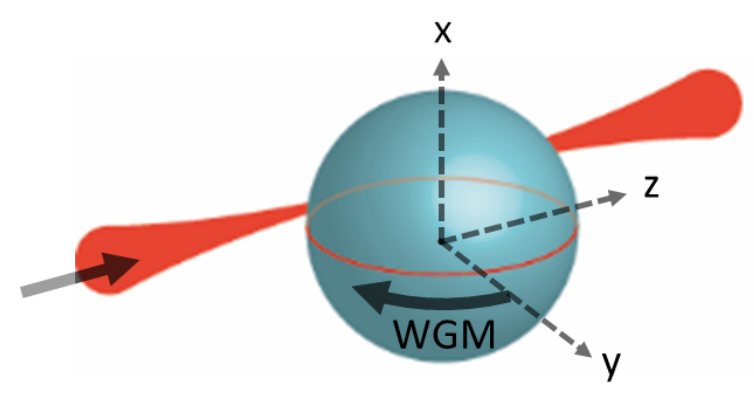

Figure 1. Schematics of a direct WGM excitation. An incident beam propagating along the $z$ axis is displaced a distance $d$ in the $y$ axis, and it tangentially hits the microresonator. As a result, a WGM that oscillates around the $x$ axis is excited.

mogeneous sphere embedded in a homogeneous and nonabsorbing medium. The sphere has an index of refraction $n_{r}$ and a radius $R$. The sphere is illuminated by a focused Gaussian beam with a well-defined helicity [7] at a transverse distance $d$ from the center of the sphere (see Figure 1). The EM field is decomposed in three sub-fields: the incident $\mathbf{E}^{\mathrm{i}}$, the scattered $\mathbf{E}^{\text {sca }}$ and the interior $\mathbf{E}^{\text {int }}$. All the fields are decomposed into multipolar fields whose origin is in the center of the sphere [7]. We express the multipolar basis as $\left\{\mathbf{A}_{j m_{z}}^{(y)}\right\}$, with $(y)=(e)$ being the electric multipoles and $(y)=(m)$ the magnetic ones. Due to the symmetries of the problem, the analytical expressions of $\mathbf{E}^{\text {sca }}$ and $\mathbf{E}^{\text {int }}$ are readily obtained when the expression of $\mathbf{E}^{\mathrm{i}}$ is known. The expression of focused Gaussian beam with a well-defined helicity propagating along the $z$ axis at a distance $\mathbf{d}$ of the 


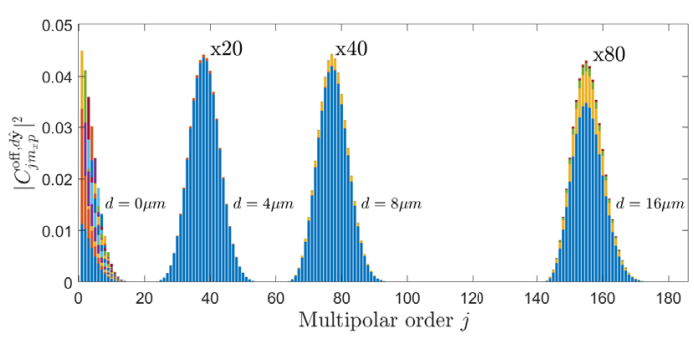

Figure 2. Displaced beam shape function $\left|C_{j, m_{x}, p}^{\text {off, } d \hat{\mathbf{y}}}\right|^{2}$ for the rotated multipoles $\mathbf{A}_{j m_{x}}^{(y)}$. The plots are done for $\mathrm{NA}=0.4$ and $d=0,4,8,16 \mu \mathrm{m}$. The blue color displays the weight of the multipolar modes with $m_{x}=j$, the yellow color shows the weights of $m_{x}=j-1$, etc. The plot with $d=0 \mu \mathrm{m}$ corresponds to the on-axis beam shape function with $m_{z}^{*}=1$. The wavelength is $\lambda=640 \mathrm{~nm}$.

center of the particle is given by [5]:

$$
\mathbf{E}^{\mathrm{i}}(\mathbf{d})=\sum_{j=1}^{\infty} \sum_{m_{z}=-j}^{j} C_{j, m_{z}, p}^{\mathrm{off}, \mathbf{d}}\left(\mathbf{A}_{j m_{z}}^{(e)}+p \mathbf{A}_{j m_{z}}^{(m)}\right)
$$

where the expression of $C_{j, m_{z}, p}^{\mathrm{off}, \mathbf{d}}$ is given in $[9,10]$. The most common configuration in order to excite WGMs with direct scattering can be seen in Figure 1 , where $\mathbf{d}=d \hat{\mathbf{y}}$. In order to make the calculations simpler and the simulations faster, we particularize $\mathbf{d}=d \hat{\mathbf{y}}$ and express Eq.(1) in the TAM basis. For that, we do a rotation of $-\pi / 2$ around the $y$ axis. This rotation is done with the Wigner matrix, yielding the following final expression:

$$
\begin{aligned}
\mathbf{E}^{\mathrm{i}}(d \hat{\mathbf{y}})= & \sum_{j=1}^{\infty} \sum_{m_{x}=-j}^{j} C_{j, m_{x}, p}^{\mathrm{off}, d \hat{\mathbf{y}}}\left(\mathbf{A}_{j m_{x}}^{(e)}+p \mathbf{A}_{j m_{x}}^{(m)}\right) \\
C_{j, m_{x}, p}^{\mathrm{off}, d \hat{\mathbf{y}}}= & \sum_{n=-\min \left(j, j^{\prime}\right)}^{\min \left(j, j^{\prime}\right)} \sum_{L=0}^{\infty}(2 L+1)(-i)^{L} j_{L}(k d) \\
& \left\langle j, n ; L, 0 \mid j^{\prime}, n\right\rangle\left\langle j, p ; L, 0 \mid j^{\prime}, p\right\rangle \\
& \sum_{j^{\prime}=m_{z}^{*}}^{\infty} \sum_{m_{z}=-j}^{j} \mathrm{~d}^{j}(-\pi / 2)_{m_{z}}^{m_{x}} D^{j}(\hat{\mathbf{d}})_{n}^{m_{z}} D^{j^{\prime}}(\hat{\mathbf{d}})_{n}^{m_{z}^{*}} C_{j^{\prime} m_{z}^{*} p}^{\mathrm{on}}
\end{aligned}
$$

with $j_{L}(k d)$ being the spherical Bessel function; $\left\langle j_{1}, m_{1} ; j_{2}, m_{2} \mid J, M\right\rangle$ a Clebsch-Gordan coefficient; and $D^{j}(\hat{\mathbf{r}})_{n}^{m}$ being the Wigner matrix. The analytical expression of $C_{j, m_{r}, p}^{\mathrm{off}, d \hat{\mathbf{y}}}$ given by Eq.(3) is the main theoretical result in [5]. It shows us how the multipolar decomposition of a focused Gaussian beam aligned with respect to the center of a sphere $C_{j^{\prime} m_{z}^{*} p}^{\text {on }}$ is transformed by means of a translation along the $y$ axis. The net result of such transformation is that of i) shifting the multipolar decomposition towards larger $j$ 's by an amount which is proportional to $2 \pi d / \lambda$, and ii) turning the initial AM along the $z$ axis into transverse AM along the $x$ axis [8]. This can be observed in Figure 1, where different multipolar decompositions are plotted for different $d=0,4,8,16 \mu \mathrm{m}$. Note that the expression of $C_{j^{\prime} m_{z}^{*} p}^{\text {on }}$ for a focused Gaussian beam with a well-defined helicity can be found in some previous works [7, 11-13].

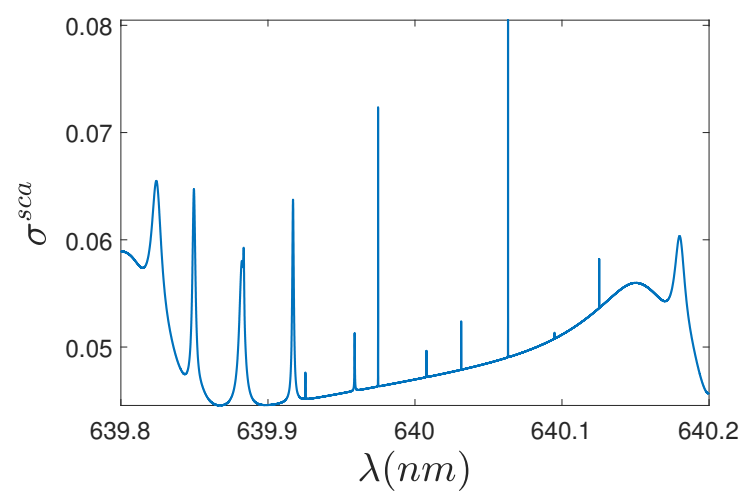

Figure 3. Scattering cross section $\sigma^{s c a}$ as a function of $\lambda$ for a sphere with $R=145 \mu \mathrm{m}$, and $n_{r}=1.3989$. The sphere is illuminated with a displaced focused Gaussian beam with a welldefined helicity. The beam is displaced $d=150 \mu \mathrm{m}$ and the lens has an NA $=0.4$.

\section{Results}

Thanks to the semi-analytical formulation given by Eqs.(2-3), we have been able to analytically compute the total EM field around a sphere with a radius $R=145 \mu \mathrm{m}$, and a relative index of refraction $n_{r}=1.3989$. The sphere is tangentially illuminated with a focused Gaussian beam with a well-defined helicity (or handedness). The focusing aplanatic lens has a numerical aperture NA $=0.4$, and it focuses the beam at a distance $d=150 \mu \mathrm{m}$ of the center of sphere. In order to mimic a scattering experiment, first we compute the scattering cross section as a function of the wavelength, for a wavelength scan of $\Delta \lambda=0.4 \mathrm{~nm}$ centred at $\lambda=640 \mathrm{~nm}$. In Figure 3, we observe that a very narrow $\lambda$ scan of $\Delta \lambda=0.4 \mathrm{~nm}$ is enough to find many resonances. Here, we are interested in studying the coupling efficiency (CE) as well as the mode purity (MP) of one of these resonances. We will study the resonance that maximizes $\sigma^{s c a}$ in this range, which happens at $\lambda=640.063295$ $\mathrm{nm}$. Our choice is motivated by the experimental measurements, where a greater scattering signal will make the measurement of CE easier. Thanks to our semi-analytical code, we can find the fundamental mode which is causing the sphere to resonate at this wavelength: in this case, it is the magnetic Mie coefficient $b_{1456}$. However, not all the scattered power is given by this mode. Some other modes also give some partial contributions to the scattering. We can quantify the purity of a resonant mode by computing its contribution to the total scattering. Specifically, we compute the MP as the scattered power given by a targeted single mode (the WGM given by $b_{1456}$ in this case), divided by the total scattered power. Analytically, it 


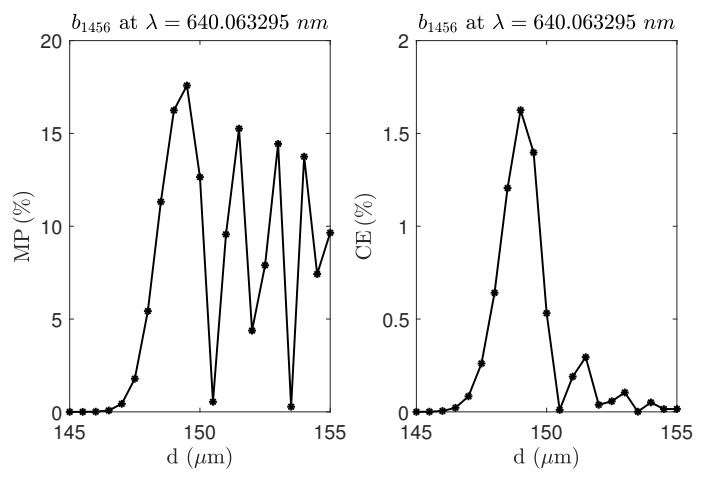

Figure 4. Mode Purity (MP) and Coupling Efficiency (CE) as a function of the transverse position $d$ for a WGM defined by the magnetic Mie coefficient $b_{1456}$. The spherical resonator has $R=$ $145 \mu \mathrm{m}$ and $n_{r}=1.3989$. The mode is excited at $\lambda=640.063295$ $\mathrm{nm}$.

can be expressed as:

$$
\begin{aligned}
\operatorname{MP}_{j^{*}}^{(e)}(d) & =\frac{\left|a_{j^{*}} C_{j^{*}, m_{x}=j^{*}, p}^{\mathrm{off}, \hat{\mathbf{y}}}\right|^{2}}{\sum_{j=1}^{\infty} \sum_{m_{x}=-j}^{j}\left|C_{j, m_{x}, p}^{\mathrm{off}, d \hat{\mathbf{y}}}\right|^{2}\left(\left|a_{j}\right|^{2}+\left|b_{j}\right|^{2}\right)} \\
\operatorname{MP}_{j^{*}}^{(m)}(d) & =\frac{\left|b_{j^{*}} C_{j^{*}, m_{x}=j^{*}, p}^{\mathrm{off}, d \hat{\mathbf{y}}}\right|^{2}}{\sum_{j=1}^{\infty} \sum_{m_{x}=-j}^{j}\left|C_{j, m_{x}, p}^{\mathrm{off}, d \hat{\mathbf{y}}}\right|^{2}\left(\left|a_{j}\right|^{2}+\left|b_{j}\right|^{2}\right)}
\end{aligned}
$$

where Eq.(4) quantifies the MP of electric modes, and Eq.(5) does the same for magnetic modes. We have used Eq.(5) to compute the MP of the magnetic mode with $j^{*}=1456$ resonating at $\lambda=640.063295 \mathrm{~nm}$, as a function of the displacement $d$. The theoretical results are shown in Fig.4. We observe that despite measuring the MP at the resonant wavelength of the $b_{1456}$ mode, the values can oscillate in between $0-20 \%$. It is clear that the transverse position of the beam $d$ places a crucial role regarding the excitation of a pure mode. In contrast, the CE is computed as the total scattered power given by a targeted single mode, divided over the total incident power. Analytically, we can express it as:

$$
\begin{aligned}
\mathrm{CE}_{j^{*}}^{(e)}(d) & =\left|a_{j^{*}} C_{j^{*}, m_{x}=j^{*}, p}^{\mathrm{off}, \hat{\mathrm{y}}}\right|^{2} \\
\mathrm{CE}_{j^{*}}^{(m)}(d) & =\left|b_{j^{*}} C_{j^{*}, m_{x}=j^{*}, p}^{\text {off } d \hat{\mathbf{y}}}\right|^{2}
\end{aligned}
$$

where the function $C_{j, m_{x}, p}^{\mathrm{off}, d \hat{\mathbf{y}}}$ needs to be such that the incident power is normalized to 1 :

$$
\sum_{j=1}^{\infty} \sum_{m_{x}=-j}^{j}\left|C_{j, m_{x}, p}^{\mathrm{off}, d \hat{\mathbf{y}}}\right|^{2}=1
$$

The theoretical results of the CE computed using Eq.(7) can also be seen in Figure 4. We observe that the CE is much more sensitive to the transverse distance $d$ than the MP. It can also be observed that both MP and CE peak at different $d$ values. Also, note that the peak of both MP and CE happen for a value of $d$ which is larger than the radius of the particle. That is, it is clear that the excitation of

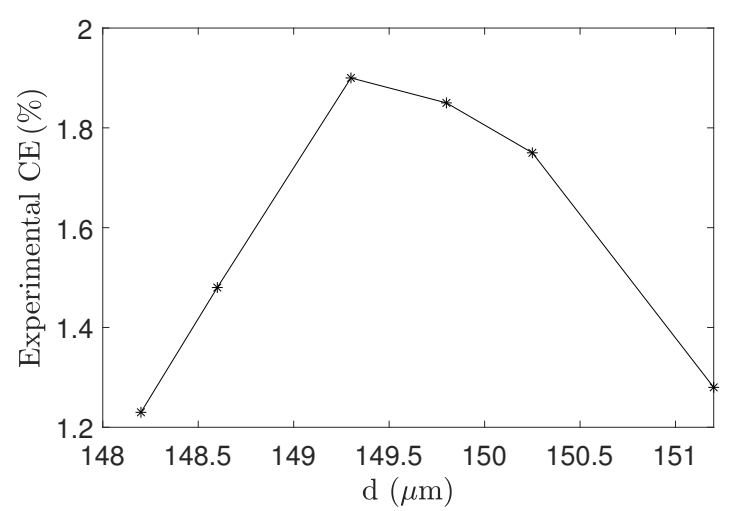

Figure 5. Experimental CE for an equivalent $b_{1456}$ mode as a function of the transverse distance $d$. The pendant droplet has a radius of $R=156.5 \mu \mathrm{m}$, and it is made of silicone oil with $n_{r}=1.3989$. The laser is works at $640 \mathrm{~nm}$.

WGM needs to be done a position $d$ such that most of the beam does not directly hit the surface of the sphere. Taking all these theoretical aspects into account, we have experimentally verified that the CE retrieved from our theoretical model matches the experimental conditions. In order to do that, we have measured the backscattering of a spherical pendant droplet made of silicone oil with $n_{r}=1.3989$. The radius of the pendant droplet has been measured to be $R=156.5 \mu \mathrm{m}$, and the wavelength of the laser is centred at $\lambda=640 \mathrm{~nm}$. The laser beam is focused with a lens with an NA $=0.4$. The wavelength scans are done by varying the temperature of the diode laser. We have studied a resonance with a $\mathrm{Q}$ factor of the same order of magnitude as the one given by the Mie mode $b_{1456}$. We experimentally determine the $\mathrm{CE}$ as the ratio between the resonantlycoupled optical power and incident optical power. In Figure 5, we plot the measured CE for the pendant droplet. It can be seen that the matching between the experimental value and the simulated one is outstanding. Not only the absolute values are very similar, but also the functional shape of $\mathrm{CE}$ as a function of $d$ is almost equal. Besides, we observe that both the experimental and theoretical $\mathrm{CE}(\%)$ is of the order of $2 \%$. Note that this value is clearly within the boundaries of a theoretical threshold calculated in a previous work [14].

\section{Conclusion}

We have developed an analytical theoretical method that quantitatively predicts the wavelength, Q-factor, coupling efficiency and mode purity of WGMs excited with tangentially free propagating beam. Thanks to this model, we have demonstrated that the efficient excitation of WGMs with free propagating beams needs to be performed at transverse distances $d$ which are greater than $R$. We have computed the MP of a resonant $b_{1456} \mathrm{WGM}$, and have seen that its value is below $20 \%$. We have also observed that the transverse distance $d$ has a huge impact in the MP, varying it from 0 to nearly $20 \%$ for the same resonant wavelength. Finally, we have also compared the experi- 
mental and theoretical CE for a $300 \mu \mathrm{m}$ resonator, yielding very similar results, of the order of $2 \%$. We foresee that our method can be applied both to understand current experimental undertakings and to predict new effects.

\section{References}

[1] A. Chiasera, Y. Dumeige, P. Feron, M. Ferrari, Y. Jestin, G. Nunzi Conti, S. Pelli, S. Soria, G.C. Righini, Laser \& Photonics Reviews 4, 457 (2010)

[2] L. He, Ş.K. Özdemir, L. Yang, Laser \& Photonics Reviews 7, 60 (2013)

[3] A. Giorgini, S. Avino, P. Malara, P. De Natale, G. Gagliardi, Sensors 19, 473 (2019)

[4] D. D’Ambrosio, M. Capezzuto, S. Avino, P. Malara, A. Giorgini, P. De Natale, G. Gagliardi, Optics Letters 46, 3111 (2021)

[5] X. Zambrana-Puyalto, D. D’Ambrosio, G. Gagliardi, Laser \& Photonics Reviews 15, 2000528 (2021)
[6] G. Gouesbet, G. Gréhan, Generalized Lorenz-Mie Theories (Springer, 2011)

[7] X. Zambrana-Puyalto, X. Vidal, G. Molina-Terriza, Opt. Express 20, 24536 (2012)

[8] I. Fernandez-Corbaton, X. Zambrana-Puyalto, N. Bonod, C. Rockstuhl, Physical Review A 94, 053822 (2016)

[9] W.K. Tung, Group Theory in Physics (World Scientific, Singapore, 1985)

[10] D.W. Mackowski, Journal of Quantitative Spectroscopy and Radiative Transfer 109, 770 (2008)

[11] X. Zambrana-Puyalto, G. Molina-Terriza, J. Quant. Spectrosc. Radiat. Transf. 126, 50 (2013)

[12] X. Zambrana-Puyalto, X. Vidal, M.L. Juan, G. Molina-Terriza, Opt. Express 21, 17520 (2013)

[13] X. Zambrana-Puyalto, X. Vidal, P. Woźniak, P. Banzer, G. Molina-Terriza, ACS Photonics 5, 2936 (2018)

[14] C.L. Zou, F.J. Shu, F.W. Sun, Z.J. Gong, Z.F. Han, G.C. Guo, Optics express 21, 9982 (2013) 